Case Report

\title{
Salvage after Retroperitoneal Kidney Allograft Torsion
}

\author{
Justin M. Greco $\mathbb{D}$, David C. Mulligan, and Peter S. Yoo \\ Division of Transplantation, Department of Surgery, Yale University School of Medicine, New Haven, CT, USA \\ Correspondence should be addressed to Justin M. Greco; justin.greco@yale.edu
}

Received 30 June 2019; Accepted 16 December 2019; Published 14 January 2020

Academic Editor: Piero Boraschi

Copyright (c) 2020 Justin M. Greco et al. This is an open access article distributed under the Creative Commons Attribution License, which permits unrestricted use, distribution, and reproduction in any medium, provided the original work is properly cited.

\begin{abstract}
Torsion of a transplanted kidney into the retroperitoneal space is a rare occurrence, with only three other reported cases. Failure after kidney transplantation is caused by surgical, immunological, and infective complications. Torsion is a complication that poses a serious risk of ischemic graft failure, and, if suspected, sonographic evaluation helps ascertain the diagnosis. Here, we present the case of a 69-year-old transplant recipient whose routine postoperative ultrasound confirmed vessel patency, however subsequently developed clinical signs of renal allograft compromise. Repeat ultrasound showed signs of vascular compromise and the patient was emergently re-explored. Torsion of the renal allograft about its pedicle was encountered and corrected by manual detorsion and nephropexy to the retroperitoneal wall. Clinicians should recognize pedicle torsion as a potential cause of renal allograft failure and the role of nephropexy in its management.
\end{abstract}

\section{Introduction}

A total of 19,128 kidney transplants were performed in 2016, and allograft failure is a devastating complication following transplant [1]. Early postoperative causes of allograft loss result from acute rejection, renal vein thrombosis, or renal artery stenosis. Torsion of kidneys transplanted into the retroperitoneal space is a rare complication. Only three other cases have been reported, all within the last ten years [2-4]. These cases represent examples of warm ischemic time in kidney transplants. Torsion is not uncommon after intraperitoneal transplantation, with cases being documented in the setting of simultaneous pancreas and kidney transplants [5-7]. The presumed causative factors are length of the allograft vein, artery, and ureter; location in the peritoneal space; and torque forces of surrounding organs. In contrast, the tight retroperitoneal space should naturally prevent twisting of the renal allograft. Nonetheless, clinical suspicion for this complication and early intervention are critical to salvage a transplanted kidney in any case where vascular compromise is implicated.

\section{Case Presentation}

A 69-year-old woman with autosomal dominant polycystic kidney disease (ADPKD), two years of peritoneal dialysis and two years of hemodialysis, underwent a donation after brain death kidney transplant. ADPKD had affected her mother, maternal grandmother, aunt, and uncle. In addition to renal failure, the patient's medical problems included hypertension, secondary hyperparathyroidism, and arthritis. Her calculated panel of reactive antibodies was $71 \%$. Past surgical history included tonsillectomy, adenoidectomy, appendectomy, and cholecystectomy. The patient reported rare alcohol use and a remote 0.5 pack year smoking history. The patient had a preoperative weight of $57.2 \mathrm{~kg}$ and a BMI of 25.89 .

The kidney was transplanted into the retroperitoneum via a Gibson incision in the right iliac fossa after 13 hours and 52 minutes of cold ischemic time. The graft was from a female donor, right-sided, and $11 \mathrm{~cm}$ in length. Several renal cysts in the native kidney were drained to make space for the transplant. The renal allograft pedicle consisted of a single artery, vein, and ureter; there was not an aortic patch. The 

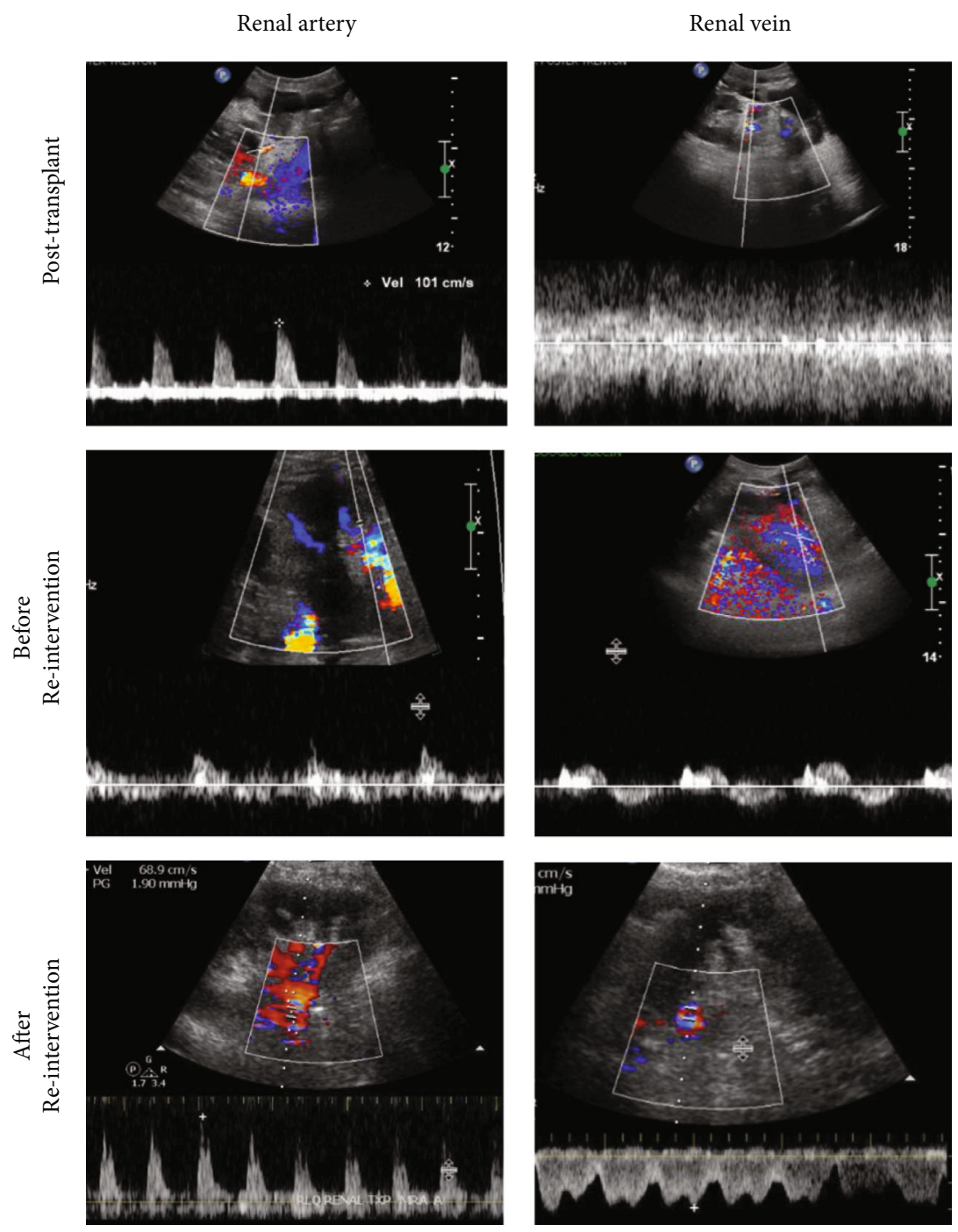

FIGURE 1: Ultrasound findings of the renal allograft vein and artery.

donor renal artery and vein were anastomosed to the recipient external iliac vessels, in an end-to-side fashion. A J-stent was placed across the uretero-vesical anastomosis. The initial intraoperative urine output was low but, substantially increased to $1270 \mathrm{~mL}$ during the first 4 hours. Preoperative serum creatinine declined from $6.51 \mathrm{mg} / \mathrm{dL}$ to $3.20 \mathrm{mg} / \mathrm{dL}$ after completion of the procedure, and the blood urea nitrogen (BUN) also decreased from $47 \mathrm{mg} / \mathrm{dL}$ to $17 \mathrm{mg} / \mathrm{dL}$. In keeping with our institution's standard of practice, an immediate allograft ultrasound was done in the post-anesthesia recovery unit which demonstrated normal flow dynamics and elevated resistive indices, with no hydronephrosis or perinephric collection (Figure 1).

Over the next 18 hours, the urine output gradually dropped to $321 \mathrm{~mL}$, at a rate less than $0.5 \mathrm{~mL} / \mathrm{kg} / \mathrm{hr}$, which did not respond to an intravenous fluid challenge. This prompted a repeat ultrasound of the renal allograft. Tardus parvus waveforms and nonvisualization of the renal vein were observed, which was concerning for arterial stenosis, arterial thrombosis, or venous thrombosis. The patient was emergently dialyzed and taken to the operating room 30 hours after the initial procedure. On re-exploration, the kidney allograft was found to be rotated 180 degrees clockwise with near total occlusion of the renal artery, vein, and ureter.

Detorsion of the kidney resulted in restored perfusion, palpable pulse, and good Doppler signals in both the artery and vein. Normal turgor and pink color returned. The allograft was then secured by a nephropexy to prevent future torsion using a superior pole silk suture and application of Arista potato starch, to induce scarring. During the next four postoperative hours, the urine output improved to $881 \mathrm{~mL}$ and the Doppler ultrasound showed good renal vein flow and absence of tardus parvus waveforms (Figure 1). In parallel, the creatinine decreased from $3.68 \mathrm{mg} / \mathrm{dL}$ to $2.60 \mathrm{mg} / \mathrm{dL}$ and BUN dropped to $25 \mathrm{mg} / \mathrm{dL}$ from $36 \mathrm{mg} / \mathrm{dL}$.

The patient was discharged on postoperative day 10 . However, she experienced further complications leading to multiple readmissions. These included ureteral stent migration and replacement, percutaneous drainage of a 


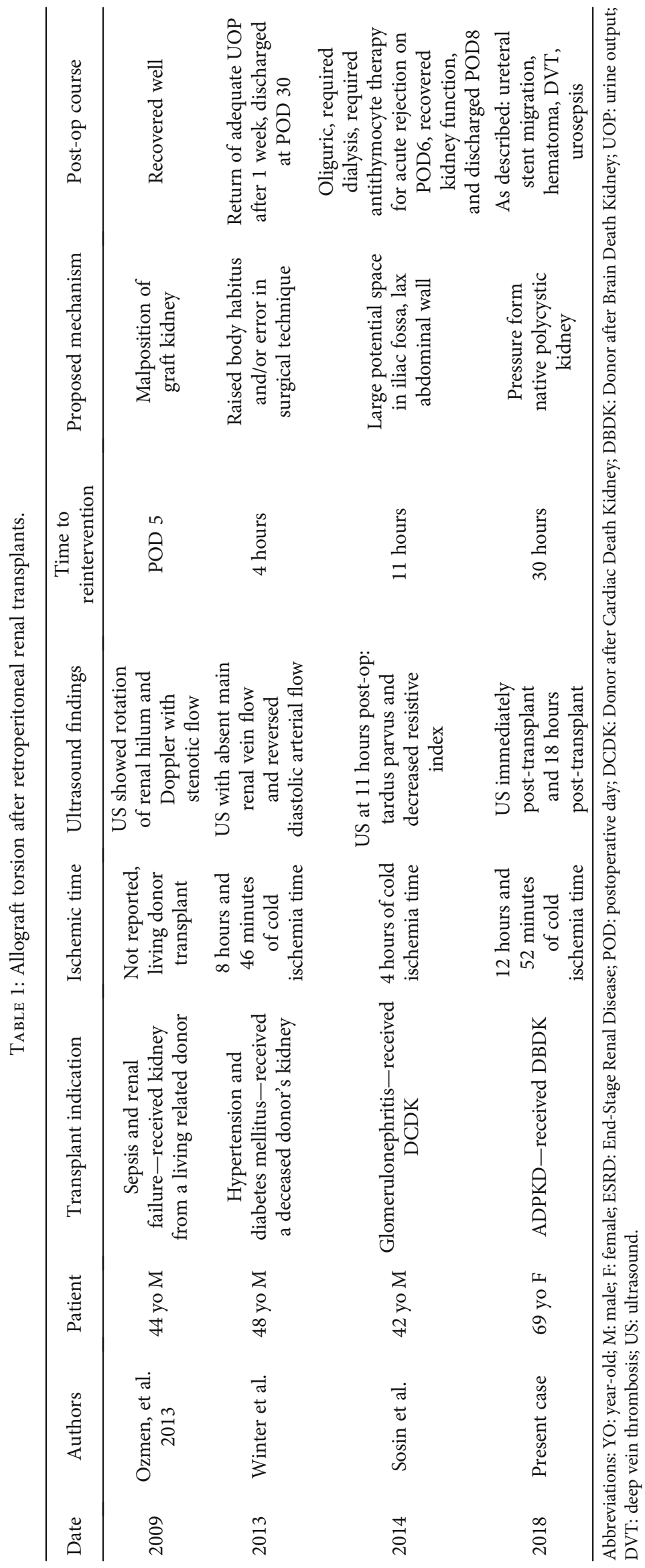


periallograft hematoma on two occasions, deep vein thrombosis requiring placement of an inferior vena cava filter, and urosepsis. The patient's last discharge was 56 days after the transplant, and she has remained well since.

\section{Discussion}

Only three other cases of retroperitoneal renal allograft torsion have been reported in the literature. In 2009, Ozmen et al. suspected malposition of the kidney when urine output dropped significantly on postoperative day 5 , leading to the identification of allograft torsion on ultrasound (Table 1) [2]. Winter et al. described a case of torsion they believe resulted from the patient's increased BMI and error in surgical technique [4]. In 2014, Sosin et al. reported laxity of the abdominal wall and accompanying potential large retroperitoneal space as the primary factors leading to rotation of the graft [3]. We believe that our case represents yet another situation whereby an enlarged native polycystic kidney exerted pressure and caused rotation of the renal allograft about its pedicle. Given this patient's BMI and small frame, torsion was further facilitated with postoperative patient movement and ambulation. The time interval to intervention in all these cases ranged from as little as 4 hours to 5 days, but in each instance, the kidney transplant was salvaged. Decreased urine output followed by sonographic postoperative evaluation demonstrating compromised arterial and venous flow prompted operative exploration. Altogether, these four cases should serve to raise awareness of this serious but reversible complication. Furthermore, transplant surgeons should consider nephropexy as a means of reducing the risk of renal allograft torsion in retroperitoneal transplants.

\section{Abbreviations}

ADPKD: Autosomal dominant polycystic kidney disease BUN: Blood urea nitrogen.

\section{Conflicts of Interest}

The authors of this paper have no conflicts of interest to declare.

\section{References}

[1] A. Hart, J. M. Smith, M. A. Skeans et al., "OPTN/SRTR 2016 Annual Data Report: Kidney," American Journal of Transplantation, vol. 18, pp. 18-113, 2018.

[2] M. M. Ozmen, I. Bilgic, I. Ziraman, and M. Koc, "Torsion of extraperitoneally transplanted kidney: an unusual complication," Experimental and Clinical Transplantation, vol. 11, no. 2, pp. 186-190, 2013.

[3] M. Sosin, W. Lumeh, and M. Cooper, "Torsion of the retroperitoneal kidney: uncommon or underreported?," Case Reports in Transplantation, vol. 2014, no. 1, Article ID 561506, 4 pages, 2014.

[4] T. C. Winter, A. L. Clarke, and J. Campsen, "Acute torsion of a retroperitoneal renal transplant mimicking renal vein thrombosis," Ultrasound Quarterly, vol. 29, no. 3, pp. 203-204, 2013.
[5] R. Dewan, A. K. Dasyam, H. Tan, and A. Furlan, "Renal allograft torsion: US and CT imaging findings of a rare posttransplant complication," Case Reports in Radiology, vol. 2016, Article ID 4273780, 3 pages, 2016.

[6] A. Lucewicz, A. Isaacs, R. D. M. Allen, V. W. T. Lam, S. Angelides, and H. C. C. Pleass, "Torsion of intraperitoneal kidney transplant," ANZ Journal of Surgery, vol. 82, no. 5, pp. 299-302, 2012.

[7] P. Kandula, A. Patel, and S. B. AMERICAN, "Torsion and inversion of intraperitoneal kidneys manifesting as acute allograft dysfunction-a case series," in American Journal of Transplantation, vol. 18, pp. 826-827, Wiley, 111 River ST, Hoboken 07030-5774, NJ USA, 2018. 


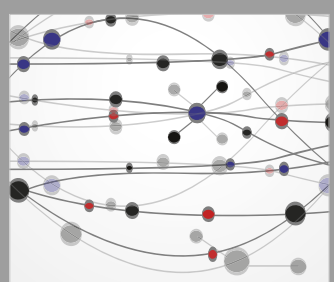

The Scientific World Journal
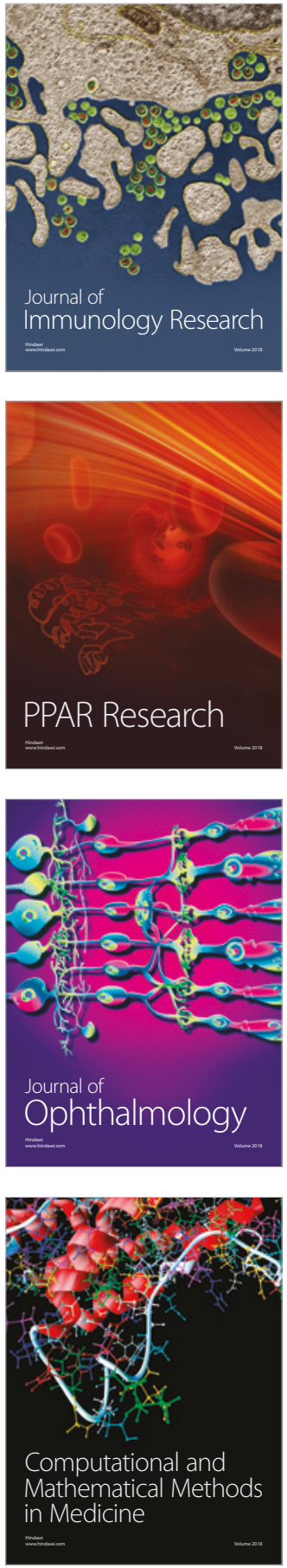

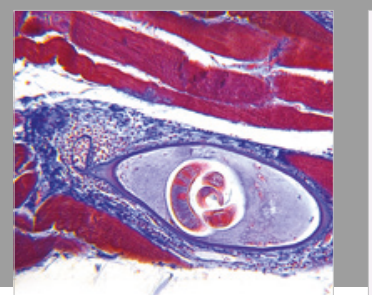

Gastroenterology Research and Practice

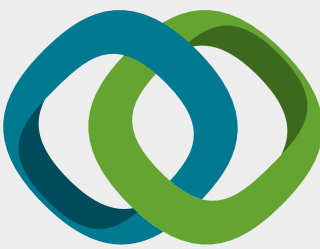

\section{Hindawi}

Submit your manuscripts at

www.hindawi.com
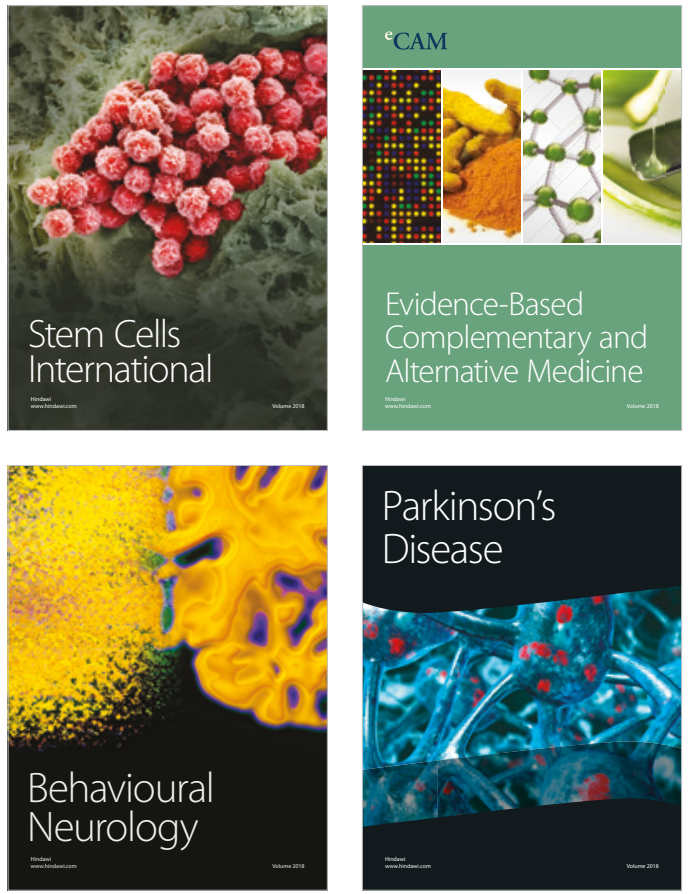

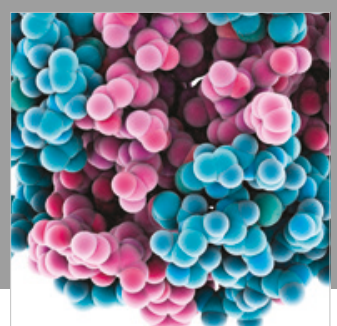

ournal of

Diabetes Research

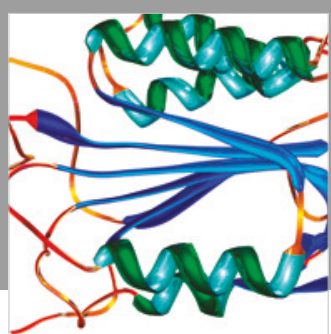

Disease Markers
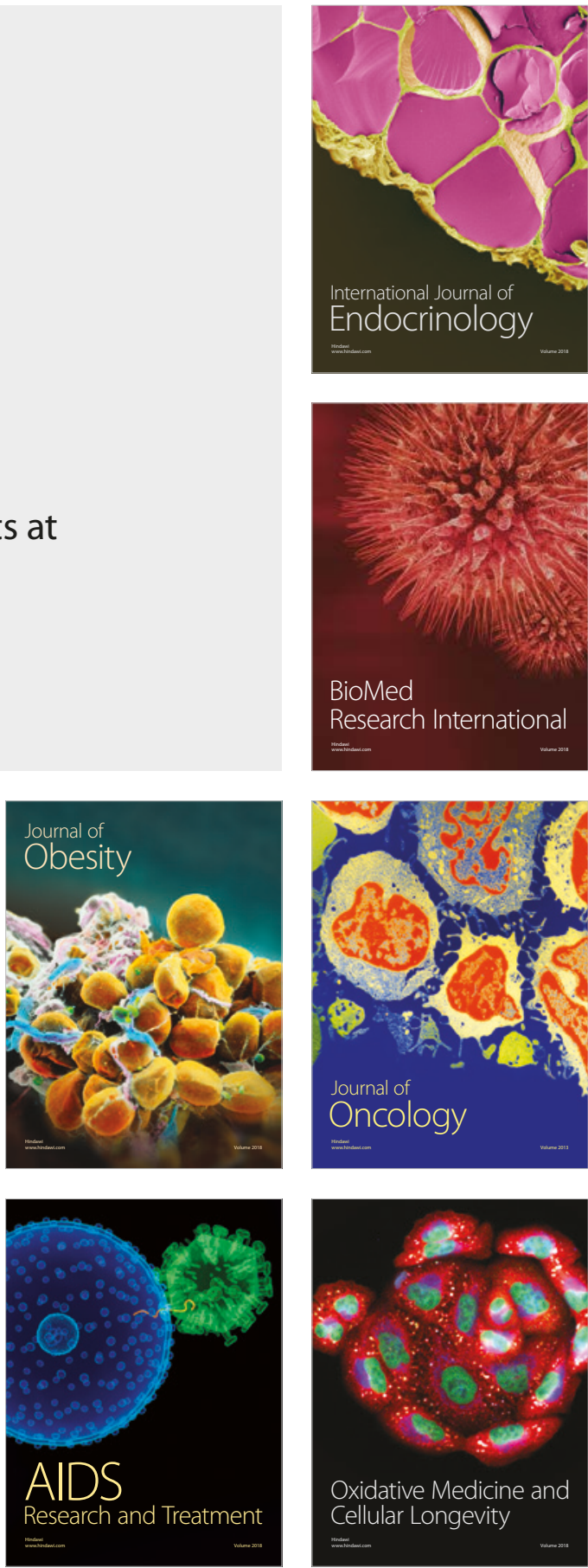